\title{
Activation of Bax in Three Models of Retinitis Pigmentosa
}

\author{
Antonella Comitato, ${ }^{1}$ Daniela Sanges $,{ }^{*}, 1$ Alessandra Rossi,${ }^{1}$ Marian M. Humphries, ${ }^{2}$ and Valeria \\ Marigo $^{1}$
}

${ }^{1}$ Department of Life Sciences, University of Modena and Reggio Emilia, Modena, Italy

${ }^{2}$ The Ocular Genetics Unit, Smurfit Institute of Genetics, Trinity College Dublin, Dublin, Ireland

Correspondence: Valeria Marigo, Department of Life Sciences, University of Modena and Reggio Emilia, via Campi 287, 41125 Modena, Italy; valeria.marigo@unimore.it

Current affiliation: *Center for Genomic Regulation (CRG), Barcelona, Spain.

Submitted: January 9, 2014

Accepted: April 27, 2014

Citation: Comitato A, Sanges D, Rossi A, Humphries MM, Marigo V. Activation of Bax in three models of retinitis pigmentosa. Invest Ophthalmol Vis Sci. 2014;55:3555-3561. DOI:

10.1167/iovs.14-13917
Purpose. The process of photoreceptor cell death in retinitis pigmentosa is still not well characterized, and identification of common mechanisms will be instrumental for development of therapeutic strategies. Here we investigated activation of Bax in $r d 1, \mathrm{P} 23 \mathrm{H}$ transgenic, and Rho knockout retinas.

Methods. Bax activation was evaluated by immunofluorescence using anti-activated Baxspecific antibodies and by Western blotting on mitochondrial protein extracts. Knockdown of cathepsin D, calpain 1, and calpain 2 was achieved by short hairpin RNA (shRNA) delivery in rd1 mutant photoreceptors cells differentiated from retinal neurospheres. The mechanism of Bax activation through calpains was evaluated in vivo by intravitreal injection of calpastatin.

Results. We defined activation and mitochondrial localization of Bax as well as activation of calpains and cathepsin $\mathrm{D}$ in the three models of retinitis pigmentosa. Taking advantage of an in vitro culture system for $r d 1$ mutant photoreceptors, we unraveled the mechanism of Bax activation. We demonstrated that calpain 1 and cathepsin D contributed to activation of Bax and to apoptosis-inducing factor (Aif) nuclear translocation. In vivo interference with calpain activity blocks Bax activation in the $r d 1$ and Rho knockout retinas and reduces activation in the $\mathrm{P} 23 \mathrm{H}$ transgenic retina.

Conclusions. Activation of Bax was observed in all three models of retinitis pigmentosa and leads to neurodamage by localization at the mitochondrion. Our data suggest that Bax can be envisaged as one of the promising target molecules for restraining photoreceptor degeneration.

Keywords: rhodopsin, rd1 mouse, $\mathrm{P} 23 \mathrm{H}$, calpastatin
$\mathrm{C}$ ell death is largely regulated by the $\mathrm{Bcl} 2$ family of interacting proteins. In mammalian cells this family of proteins is divided into three main subfamilies: prosurvival members such as $\mathrm{Bcl} 2$ and $\mathrm{Bcl}_{\mathrm{XL}}$; apoptotic $\mathrm{Bax}$ members including Bax and Bak; and apoptotic BH3-only members such as Bim, Bik, and Puma. The decision whether to live or die is often influenced by interactions among these proteins and by their relative intracellular concentration. ${ }^{1}$ Bax and Bak play central roles in apoptosis because after their activation they oligomerize at the mitochondrial outer membrane, causing its breaching and the release of apoptogenic factors including cytochrome $\mathrm{c}$ and Smac/Diablo. Whereas Bak shows constitutive mitochondrial localization, Bax is cytosolic in healthy cells and translocates to mitochondria after activation in response to apoptotic signals. Prosurvival $\mathrm{Bcl} 2$ normally inhibits $\mathrm{Bax}$ activation by direct binding, but the consequences of Bax/ $\mathrm{Bcl} 2$ interaction are still under investigation. ${ }^{2}$ Furthermore, Bax localization to the mitochondrial membrane is associated with cell death but does not necessarily cause mitochondrial outer membrane permeabilization and cytochrome c release. ${ }^{2}$

Several animal models are available for the study of retinitis pigmentosa (RP). We previously characterized the molecular mechanism leading to photoreceptor cell death in the $r d 1$ murine model bearing a loss of function of the phototransduction enzyme phosphodiesterase 6b (Pde6b). We demonstrated that influx of calcium ions into photoreceptor cells activates calpains. Calpains are proteases that cleave mitochondrial apoptosis-inducing factor (Aif) that exits from the mitochondrion and translocates to the nucleus, playing a fundamental role in rod cell death. ${ }^{3}$ Calpains appear to have a central role in the mutant $r d 1$ retina because degeneration could be restrained by exposure of the retina to calpain blockers. ${ }^{3,4}$ We previously showed that in $r d 1$ mutant rods, activation of calpains regulates subsequent mitochondrial release of Aif. $^{3}$ In different cellular systems, it has been reported that Aif is cleaved by calpains and its exit from mitochondria requires $\mathrm{Bax}$, but not $\mathrm{Bak}^{5-7}$ Bax itself can be activated by calpains and/or cathepsin $\mathrm{D}$, which are activated in rd1 degenerating rods. ${ }^{8,9}$

Previous studies showed very limited involvement of caspase 3 in the $r d 1$ mutant retina, ${ }^{10}$ although activation of effector caspases as well as calpains could be observed in a different model of RP expressing P23H mutant rhodopsin. ${ }^{11,12}$ On the other hand, no information is available concerning the molecular mechanisms of cell death in retinas lacking rhodopsin expression.

The purpose of this study was to define if Bax is activated in three murine models of retina degeneration and to evaluate whether active Bax participates in retinal degeneration in vivo. Secondly, we defined the mechanism of Bax activation in the rd1 model, taking advantage of the availability of an in vitro cell system for molecular studies of this mutation. Our data confirmed that Bax is activated in the degenerating retina and 
that a protease cascade mediates its activation. We finally show that Bax activation requires calpains in vivo.

\section{Methods}

\section{Animal Care and Treatments}

All procedures on mice were conducted at Centro Servizi Stabulario Interdipartimentale, were approved by the Ethical Committee of University of Modena and Reggio Emilia (Prot. N. 50 12/03/2010) and by Italian Ministero della Salute, and were in accordance with the ARVO Statement for the Use of Animals in Ophthalmic and Vision Research. C3H/HeN ( $r d 1), 129 \mathrm{sv}$, and C57BL6J mice were purchased from Harlan Italy (Udine, Italy). Rhodopsin $\mathrm{P} 23 \mathrm{H}$ transgenic mice $\left(\mathrm{P}_{23} \mathrm{H}^{\mathrm{Tg}}\right)^{13}$ were kindly provided by T. Dryja. Rhodopsin knockout mice ${ }^{14}$ were bred on the $129 \mathrm{sv}$ genetic background. Mice were maintained in 12-hour light/dark cycles. For intravitreal administration, mice were anesthetized with an intraperitoneal injection of 2 $\mathrm{mL} / 100 \mathrm{~g}$ body weight avertin (1.25\% [wt/vol] 2,2,2-tribromoethanol and 2.5\% [vol/vol] 2-methyl-2-butanol; Sigma, Milan, Italy). Subsequently, $0.5 \mu \mathrm{L}$ calpastatin peptide $(200-\mu \mathrm{M}$ solution with an expected final concentration in the eye of $20 \mu \mathrm{M}$; Calbiochem, Milan, Italy) was delivered intravitreously via a transscleral transchoroidal approach. Control eyes received vehicle only. Treated mice were killed 16 hours post injection.

\section{Retinal Neurosphere Culture and Differentiation}

Retinal neurospheres from adult wild-type and $r d 1$ mice were isolated and cultured as previously described. ${ }^{15}$ Retinal neurospheres were plated on extracellular matrix (Sigma)-coated coverslips, differentiated with $1 \%$ fetal bovine serum, and then processed for immunohistochemical analysis. Differentiation into rod-like cells was assessed by staining with anti-rhodopsin antibodies (1D4 and RetP1; Sigma) as previously described. ${ }^{16}$ For short hairpin RNA (shRNA) studies, cells were treated with retroviruses expressing different shRNAs at the fifth day of differentiation in the presence of polybrene. Spontaneous apoptosis in $r d 1$ mutant cells but not in wild-type cells was activated after 11 days in vitro. We performed all immunofluorescence analyses at this time point, and the data shown derived from the analysis of four slides from two independent experiments.

\section{RNA Interference (RNAi)}

Sequences beginning with an AA dinucleotide were chosen in the Bax, Calpain 1 (Capn1), Calpain 2 (Capn2), and Catbepsin D (Ctsd) mRNA, and we designed hairpin RNAi template oligonucleotides (shRNA). The control shRNA was generated from scramble sequences not targeting any known gene. Sequences targeted by shRNAs are listed in Supplementary Table S1. Retroviruses from pSUPER.retro.puro vector (OligoEngine, Seattle, WA, USA) expressing the different RNAi were prepared by transient transfection in ecotropic PhoenixECO packaging cells (ATCC, Rockville, MD, USA). Efficiencies of shRNAs were checked by infection of NIH3T3 (ATCC) cells followed by Western blotting.

\section{DNA Nick-End Labeling by TUNEL and Immunofluorescence}

Eyes were fixed, embedded in paraffin, and sectioned as previously described. ${ }^{3}$ Photoreceptors differentiated from retinal neurospheres were fixed in $4 \%$ paraformaldehyde.
Apoptotic nuclei were detected by TdT-mediated dUTP terminal nick-end labeling kit (TUNEL, fluorescein; Roche, Milan, Italy) according to the producer's protocols. Primary antibodies used were as follows: anti-Aif (1:100; Sigma), antiactive $\mathrm{Bax} 6 \mathrm{~A} 7$ (recognizes the $\mathrm{N}$-term exposed after activation but not phosphorylated $\mathrm{Bax},{ }^{17}$ 1:200; Millipore, Milan, Italy), anti-Cathepsin D (1:100; Santa Cruz Biotechnology, Heidelberg, Germany), anti-Calpain 1 (1:100; Calbiochem). Secondary antibodies were Oregon Green 488 anti-goat, Alexa Fluor 568 anti-mouse and anti-rabbit antibodies (Molecular Probes, Monza, Italy). Slides were mounted with Mowiol 4-88 (Sigma) and analyzed at an Axioskop 40 fluorescence microscope (Zeiss, Arese, Italy).

\section{Calpain Activity Assay}

Cryosections from unfixed retinas or differentiated cells grown on coverslips were incubated for 15 minutes in calpain reaction buffer (CRB: $25 \mathrm{mM}$ HEPES- $\mathrm{KOH} \mathrm{pH} 7.2,65 \mathrm{mM}$ $\mathrm{KCl}, 2 \mathrm{mM} \mathrm{MgCl}_{2}, 1.5 \mathrm{mM} \mathrm{CaCl}_{2}, 2 \mathrm{mM}$ dithiothreitol) and then exposed for 1 hour at $37^{\circ} \mathrm{C}$ to the fluorescent calpain substrate CMAC, t-BOC-Leu-Met (A6520; Invitrogen, Monza, Italy) at a final concentration of $2 \mu \mathrm{M}$. Slides were washed twice for 10 minutes each in CRB, mounted with Mowiol 4-88, and analyzed at an Axioskop 40 fluorescence microscope (Zeiss) using the filter excitation/emission 365/420.

\section{Real-Time Quantitative PCR}

Total RNA was extracted with TRIzol reagent (Life Technologies, Monza, Italy), and cDNA was synthesized using Transcriptor High Fidelity cDNA Synthesis Kit (Roche). Real-time PCR was carried out with the GeneAmp 7000 Sequence Detection System (Applied Biosystems, Milan, Italy). Primers used are listed in Supplementary Table S2. Quantification results were expressed in terms of cycle threshold (Ct). All real-time quantitative PCR reactions were run in triplicate, and the $\mathrm{Ct}$ values were averaged. The $S 26$ PCR product was used as reference marker, and data were derived from the formula $2^{-\Delta \mathrm{Ct}}$.

\section{Retinal Protein Extracts and Western Blotting Analysis}

Retinas were lysed in cold lysis buffer $(50 \mathrm{mM}$ Tris- $\mathrm{HCl} \mathrm{pH} 7.4$, $150 \mathrm{mM} \mathrm{NaCl}, 1 \%$ Nonidet P-40, 0.1\% SDS, $1 \mathrm{mM}$ EDTA, $1 \mathrm{mM}$ $\mathrm{Na}_{3} \mathrm{VO}_{4}$, protease inhibitor cocktail from Sigma) and incubated 30 minutes on ice. Tissue was disrupted with 40 strokes and centrifuged at $900 \mathrm{~g}$ for 5 minutes at $4^{\circ} \mathrm{C}$. For mitochondrialenriched extracts, tissue was homogenized in $220 \mathrm{mM}$ Dmannitol, $70 \mathrm{mM}$ sucrose, $20 \mathrm{mM}$ HEPES-KOH $\mathrm{pH} 7.2,1 \mathrm{mM}$ EDTA, $0.1 \%$ BSA, $1 \mathrm{mM} \mathrm{Na} \mathrm{VO}_{4}$, protease inhibitor cocktail (Sigma) and centrifuged at $500 \mathrm{~g}$ for 10 minutes at $4^{\circ} \mathrm{C}$. The lowspeed supernatant was centrifuged at $10,000 \mathrm{~g}$ for 10 minutes at $4{ }^{\circ} \mathrm{C}$. The resulting mitochondrial pellet was resuspended in washing buffer (250 mM sucrose, $20 \mathrm{mM}$ HEPES-KOH pH 7.4, 1 mM EDTA, $1 \mathrm{mM} \mathrm{Na} \mathrm{VO}_{4}$, protease inhibitor cocktail) and centrifuged at $10,000 \mathrm{~g}$ for 10 minutes at $4^{\circ} \mathrm{C}$. The final mitochondrial pellet was resuspended in respiration buffer (250 mM sucrose, $50 \mathrm{mM}$ HEPES $\mathrm{pH} 7.4,10 \mathrm{mM} \mathrm{KH}_{2} \mathrm{PO}_{4}, 2$ $\mathrm{mM} \mathrm{MgCl} 2,1 \mathrm{mM}$ EDTA, $1 \mathrm{mM} \mathrm{Na} \mathrm{VO}_{4}$, protease inhibitor cocktail). The purity of enriched lysates was checked by Western blotting analysis with a mitochondrial marker (anticytochrome c, 1:2000; BD Biosciences, Milan, Italy) or a cytosol marker (anti-pan-actin, 1:3000; Millipore).

Equivalent amounts of protein extracts $(50 \mu \mathrm{g})$ were used for Western blot analysis. The antibodies used for Western blotting were anti-Bax 6A7 (1:2000; Millipore), anti-Bcl2 (1:2000; Cell Signaling, Milan, Italy), anti-Calpain 1 (1:1000; 
Chemicon, Segrate, Italy), anti-Calpain 2 (1:3000; Abcam, Cambridge, UK), and anti-Cathepsin D (C-20 1:1000; Santa Cruz Biotechnology). Quantification was performed by densitometry analysis of scanned images using ImageJ software (National Institutes of Health, Bethesda, MD, USA), corrected by background, and plotted as protein/normalizing protein. Data are means \pm SD of three blots with proteins derived from three animals from two biological replicates.

\section{Statistical Analysis}

Real-time PCR data, cell counts, and densitometry analyses are shown as mean $\pm \mathrm{SD}$. Paired $t$-test analysis was performed comparing data derived from at least three different wild-type to at least three different mutant retinas.

\section{RESUlts}

\section{Activation of Bax in Three Murine Models of Retinitis Pigmentosa}

To characterize activation of $\mathrm{Bax}$ and other $\mathrm{BH} 3$ family members related to cell death, we analyzed expression at the mRNA levels and compared each mutant retina to the wild-type control matched for age. During degeneration (postnatal day [P]11) in the $r d 1$ retina $^{3}$ we observed a significant increase of proapoptotic Bax transcripts and decrease of prosurvival $\mathrm{Bcl} 2$ mRNA but no change in Bak (Fig. 1A). Similar results were obtained when we analyzed the $\mathrm{P} 23 \mathrm{H}^{\mathrm{Tg}}$ retina at the peak of cell death (P9) ${ }^{13}$ (Fig. 1B), confirming a recent study in a rat model. ${ }^{18}$ Upregulation of Bax but not of Bak was observed also in the Rho ${ }^{-1-}$ retina at the peak of rod cell death $(\mathrm{P} 45)^{14}$ but, differently from what occurred in the other models, $\mathrm{Bcl} 2$ was upregulated (Fig. 1C). We confirmed increase of Bax protein (Supplementary Fig. S1) and Bax activation using antibodies recognizing the activated form of Bax. We specifically detected activated Bax in the photoreceptor cell inner segment only in the degenerating mutant retinas (Figs. 1D-F, Supplementary Fig. S3). Activation of Bax requires a conformational change with exposure of a hydrophobic domain to allow Bax localization to the mitochondrial membrane. We therefore analyzed Bax localization at the mitochondria by Western blotting on mitochondrial-enriched protein extracts. We confirmed Bax translocation to the mitochondria in response to cell death stimuli in the $r d 1$, $\mathrm{P}^{2} 3 \mathrm{H}^{\mathrm{Tg}}$, and $\mathrm{Rho}^{-/-}$mutant retinas (Figs. 1G-I). Bax could otherwise not be detected in mitochondria extracts from wildtype retinas at all the different stages that we examined.

We also assessed if $\mathrm{Bcl} 2$ was downregulated at the protein level. We could confirm a significant reduction of $\mathrm{Bcl} 2$ in protein extracts from $r d 1$ and $\mathrm{P} 23 \mathrm{H}^{\mathrm{Tg}}$ retinas (Figs. $1 \mathrm{~J}, 1 \mathrm{~K}$ ). $B c l 2$ mRNA levels in the $\mathrm{Rho}^{-1-}$ mutant retina were upregulated but not $\mathrm{Bcl} 2$ protein (Fig. 1L), suggesting that other $\mathrm{Bcl} 2$ family members may regulate $\mathrm{Bcl} 2$ at the protein level, as previously demonstrated in T-cells. ${ }^{19} \mathrm{Bcl} 2$ reduction does not appear to be a common event in retinal degeneration. Bax activation in the degenerating retina may thus require other factors aside from loss of interaction with $\mathrm{Bcl} 2$.

\section{Activation of Calpains and Cathepsin D in Three Murine Models of Retinitis Pigmentosa}

We previously reported activation of calpains in the $r d 1$ retina. ${ }^{3,4}$ Using this in situ technique we assessed activation of calpains also in $\mathrm{P} 23 \mathrm{H}^{\mathrm{Tg}}$ retinas at the age of 10 days and in $\mathrm{Rho}^{-1-}$ retinas at the age of 45 days. We could detect calpain activity in the photoreceptor cell layer in the three models of
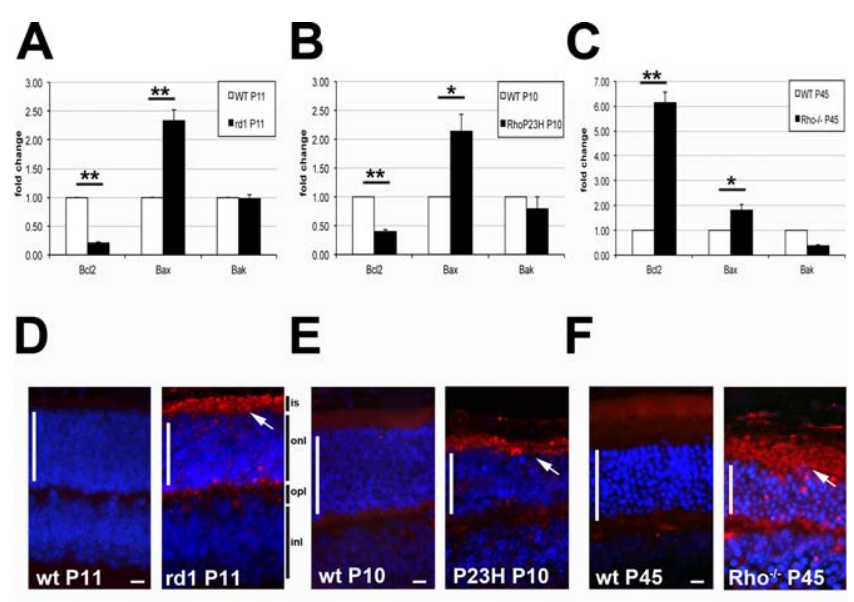

$\mathbf{F}$

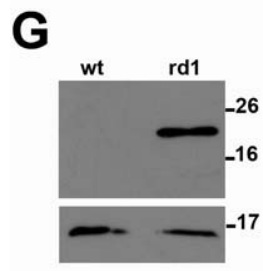

H
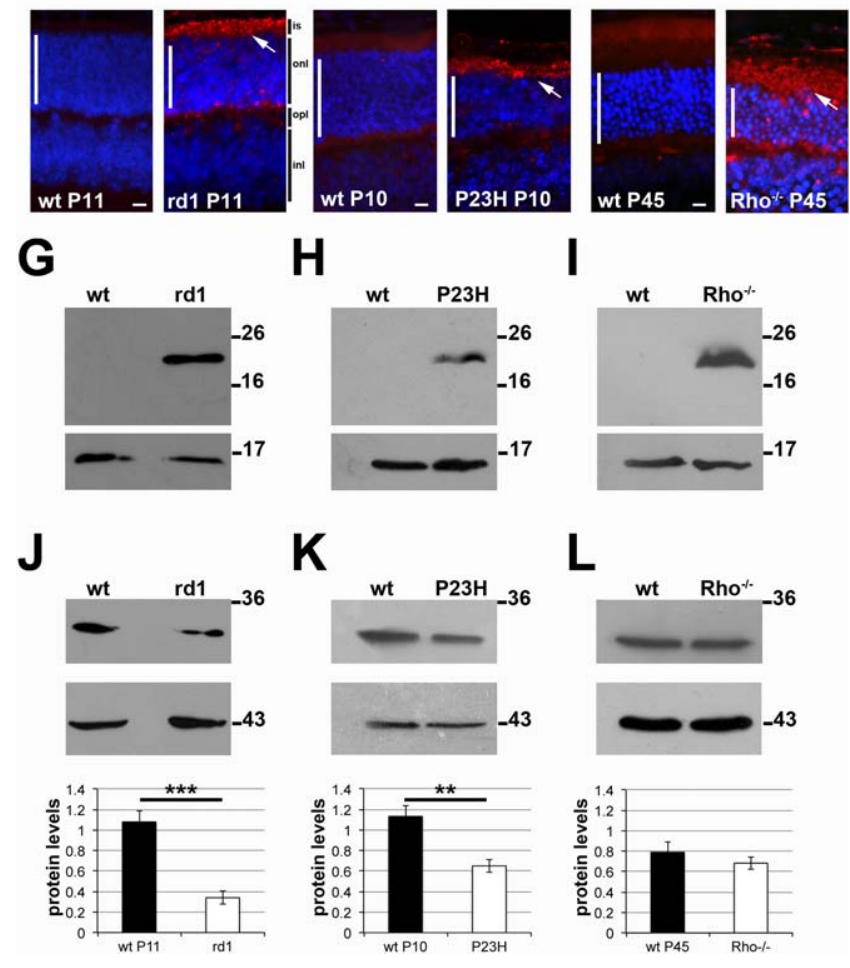

I
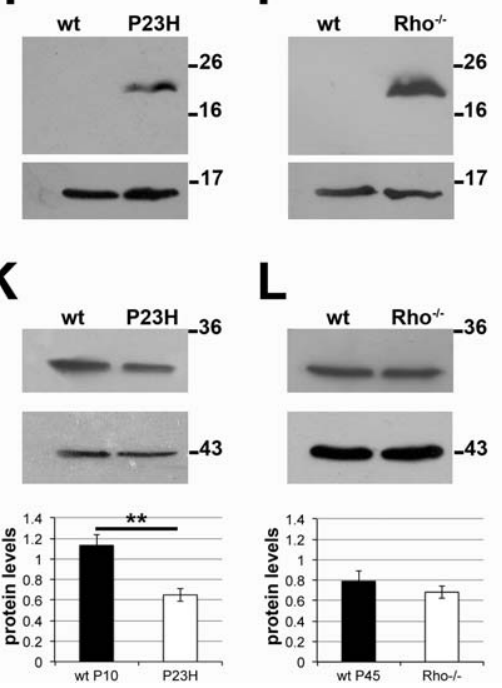

Figure 1. Bax activation during photoreceptor cell death. (A-C) Realtime PCR analysis of $\mathrm{Bcl}$, Bax, and $\mathrm{Bak}$ mRNA on P11 wild-type and rd1 retinas (A); on $\mathrm{P} 10$ wild-type and $\mathrm{P} 23 \mathrm{H}^{\mathrm{Tg}}$ retinas $(\mathbf{B})$; and on $\mathrm{P} 45$ wild-type and $\mathrm{Rho}^{-1-}$ retinas $(\mathbf{C})$. In the mutant retinas (black bars), we detected significant upregulation of Bax mRNA compared to wild-type retinas (white bars). Downregulation of $\mathrm{Bcl} 2 \mathrm{mRNA}$ was observed in $r d 1$ and $\mathrm{P} 23 \mathrm{H}^{\mathrm{Tg}}$ retinas $(\mathbf{A}, \mathbf{B})$. Upregulation of $\mathrm{Bcl} 2 \mathrm{mRNA}$ was observed in $\mathrm{Rho}^{-1-}$ retinas $(\mathbf{C})$. Values of wild-type samples were set at $1 ; n=3$. Student's $t$-test, ${ }^{* *} P \leq 0.01,{ }^{*} P \leq 0.05$. (D-F) Activation of Bax was detected with the activated Bax-specific antibody at the inner segment (arrows) of $\mathrm{P} 11$ rd1 (D), $\mathrm{P} 10 \mathrm{P} 23 \mathrm{H}^{\mathrm{Tg}}$ (E), and $\mathrm{P} 45 \mathrm{Rho}^{-1-}$ (F) retinas but not of wild-type retinas. Nuclei are stained in blue with $4^{\prime}, 6-$ diamidino-2-phenylindole (DAPI). White vertical lines indicate the layer of photoreceptor nuclei. Scale bar: $10 \mu \mathrm{m}$. (G-I) Western blotting of Bax protein in mitochondria-enriched protein extracts from P11 rd1 (G), $\mathrm{P} 10 \mathrm{P} 23 \mathrm{H}^{\mathrm{Tg}}(\mathbf{H})$, and $\mathrm{P} 45 \mathrm{Rho}^{-/}$(I) retinas and matched wild-type controls. Normalization was performed with anti-cytochrome $c$ antibodies (lower). (J-L) Western blotting of $\mathrm{Bcl} 2$ in total protein extracts from P11 rd1 (J), P10 $\mathrm{P} 23 \mathrm{H}^{\mathrm{Tg}}(\mathbf{K}), \mathrm{P} 45 \mathrm{Rho}^{-/-}(\mathbf{L})$, and the corresponding wild-type controls. Normalization was performed with anti-actin antibodies (lower). Representative Western blot (top) and quantification by densitometry (bottom) are shown. Molecular weights are shown in $\mathrm{kDa} ; n=3$. is, inner segment (containing photoreceptor cytoplasm and mitochondria); onl, outer nuclear layer; opl, outer plexiform layer; inl, inner nuclear layer.

RP but not in wild-type controls (Figs. 2A-C). By double labeling with the fluorescent calpain substrate and TUNEL, we determined that active calpains colabeled approximately $70 \%$ of cells undergoing cell death in $r d 1$ and $\mathrm{Rho}^{-/-}$mutant retinas and $50 \%$ in $\mathrm{P}_{2} 3 \mathrm{H}^{\mathrm{Tg}}$ retinas (Fig. 2D, Supplementary Fig. S2). 
A

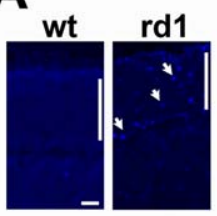

B
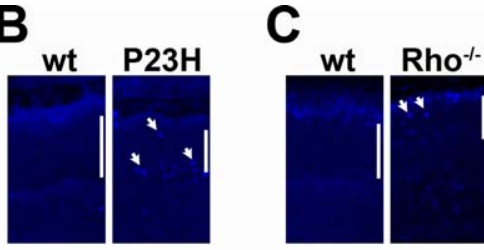

D

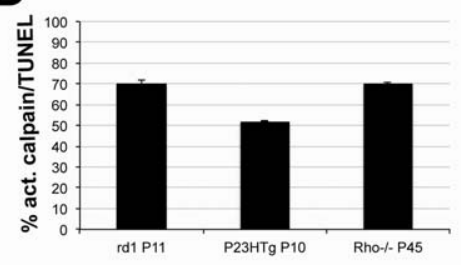

E

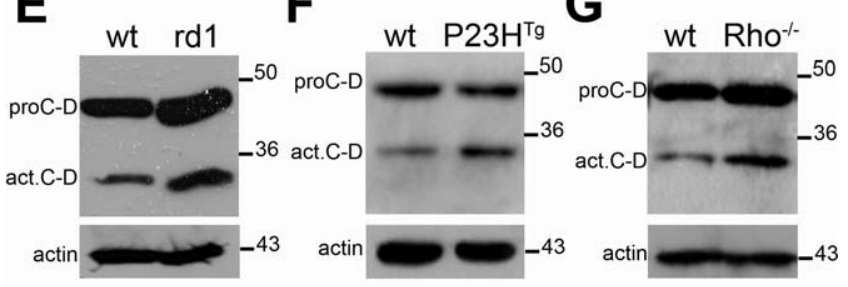

H

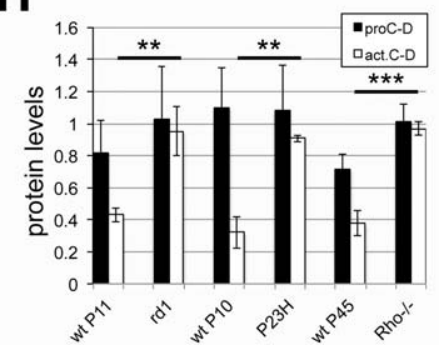

Figure 2. Calpain and cathepsin D activation during photoreceptor cell death. (A-C) We investigated calpain activity by exposure of frozen nonfixed sections to a fluorescent calpain substrate and detected several cells with high calpain activity (fluorescent spots in the outer nuclear layer, arrows) in mutant retinas but no calpain activity in the corresponding wild-type retinas. White vertical lines indicate the layer of photoreceptor cells. Scale bars: $10 \mu \mathrm{m}$. (D) Histogram representing the percentage of cells showing calpain activation among the cells undergoing cell death $\left(\mathrm{TUNEL}^{+}\right)$. In the $r d 1$ and $\mathrm{Rho}^{-1-}$ retinas, approximately $70 \%$ of the dying cells activate calpain; in the $\mathrm{P} 23 \mathrm{H}^{\mathrm{Tg}}$ retina, only $50 \% ; n=3$. (E-G) Western blotting of cathepsin D in total protein extracts from $\mathrm{P} 11 \mathrm{rd} 1$ (E), $\mathrm{P} 10 \mathrm{P} 23 \mathrm{H}^{\mathrm{Tg}}(\mathbf{F}), \mathrm{P} 45 \mathrm{Rho}^{-/}(\mathbf{G})$, and the corresponding wild-type controls. The antibody recognizes both cathepsin D (pro-C-D) and activated cathepsin D (actC-D). Normalization was performed with anti-actin antibodies (lower). Molecular weights are shown in $\mathrm{kDa}$. (H) Quantification by densitometry of Western blotting shown in (E-G). A significant increase of activated cathepsin D (white bars) was observed in the three models of retinal degeneration; $n=3$. Student's $t$-test, ${ }^{* * * *} P \leq 0.001$, ${ }^{* * *} P \leq 0.01$ comparing activated cathepsin $\mathrm{D}$ in mutant and wild-type samples

Calpain activation therefore strongly correlated with cell death in $r d 1$ and $\mathrm{Rho}^{-/-}$mice. Calpains can directly activate Bax or activate other proteases, such as cathepsin D, to trigger activation of Bax. $^{7}$ We thus assessed cathepsin $\mathrm{D}$ activation by Western blotting. In the $r d 1$ retina we confirmed activation of cathepsin D by the observation of a significant increase of the 32-kDa cathepsin D active form (Figs. 2E, 2H). Similar results were obtained analyzing protein extracts from $\mathrm{P} 23 \mathrm{H}^{\mathrm{Tg}}$ retinas at the age of 10 days and from $\mathrm{Rho}^{-/-}$retina at the age of 45 days (Figs. 2F-H).
A

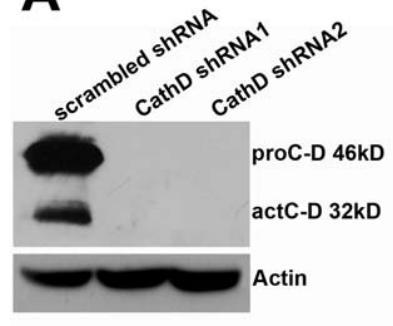

B
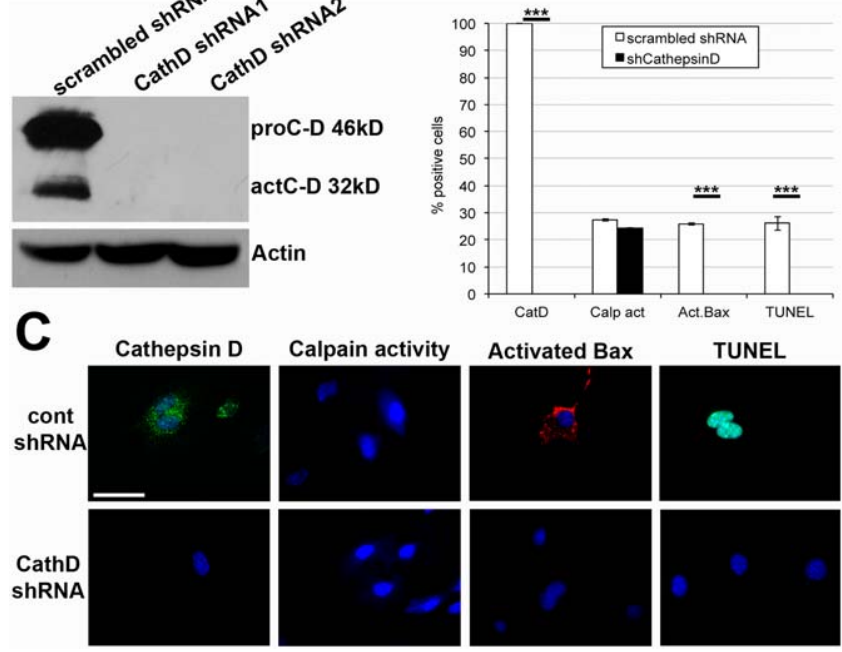

TUNEL

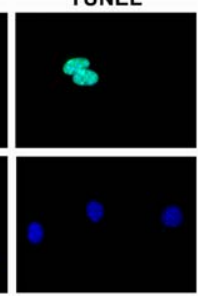

FiguRE 3. Cathepsin D regulates activation of Bax in rd1 dying photoreceptors. (A) NIH3T3 cells were infected with retroviruses expressing two shRNAs targeting Cathepsin D mRNA (Cath shRNA) or with a retrovirus expressing an shRNA that recognizes a scrambled sequence (scrambled shRNA). Western blotting was performed on total protein extracts from infected NIH3T3 cells and normalized with antiactin antibodies (lower). (B) Downregulation of Cathepsin D in rod-like cells in vitro differentiated from retinal neurospheres. Data represent percentages of positive cells labeled by immunofluorescence; $n=5$. Similar results were obtained with a second shRNA targeting a different region of Cathepsin D mRNA. (C) Representative immunofluorescence images of data shown in (B). Cathepsin D and TUNEL are shown in green, activated Bax in red; nuclei are stained in blue with DAPI. Calpain activity was investigated by exposure of cells to a blue fluorescent calpain substrate visible in cells treated with a control shRNA as well as with an shRNA targeting Cathepsin D, suggesting that Cathepsin D does not affect calpain activity. No DAPI staining was performed on slides treated with calpain substrate. Scale bar for all figure parts is shown at upper left and is $50 \mu \mathrm{m}$

Taken together, these data suggested that Bax was activated in the three models of RP and that at the time of its stimulation two classes of proteases, calpains and cathepsin D, were also active.

\section{Bax Is Activated Through Cathepsin D and Calpains in rd1 Mutant Photoreceptors}

We previously validated an in vitro model for the study of rod cell death caused by mutations in Pde6b. ${ }^{3}$ This in vitro model is based on the generation of retinal neurospheres from $r d 1$ eyes and differentiation of retinal neurospheres into rod photoreceptors. ${ }^{15}$ In the in vitro model we could detect calcium influx, calpain activation, and Aif and caspase 12 nuclear translocation correlating to cell death, similarly to the in vivo rd1 retina. ${ }^{3}$ We took advantage of this in vitro system to molecularly characterize Bax activation in $r d 1$ mutant photoreceptors. The in vitro model allowed us to knock down cathepsin $\mathrm{D}$ by shRNAs. We tested two shRNAs targeting different regions of CathD mRNA (Supplementary Table S1), and we used a retroviral system to allow $100 \%$ transduction of photoreceptor cells, as previously reported. ${ }^{3}$ Both shRNAs were very efficient in downregulation of cathepsin $\mathrm{D}$ as demonstrated at the protein level by Western blotting (Fig. 3A). We observed that knockdown of cathepsin D did not affect calpain activity but blocked Bax activation. Loss of cathepsin D could also protect from cell death (Figs. 3B, 3C). 
A
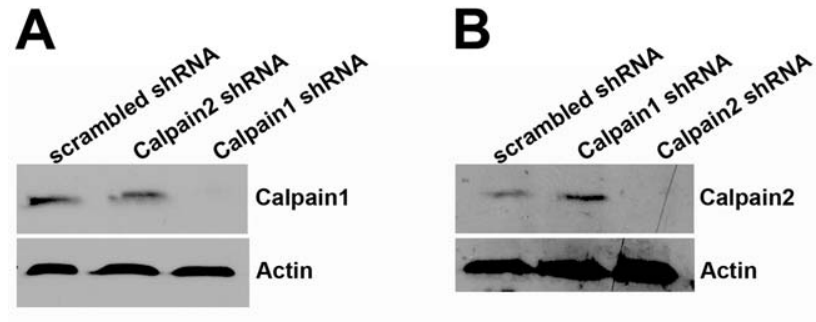

C

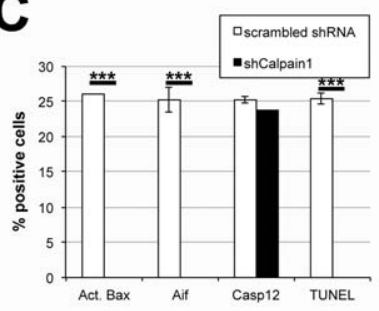

D

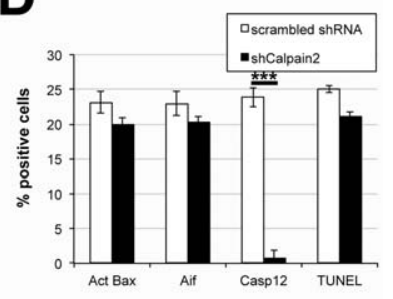

E

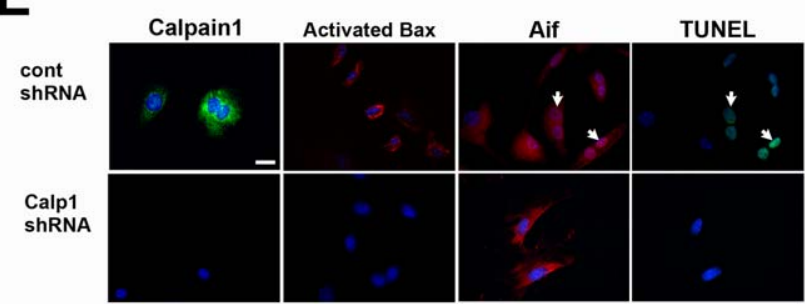

Figure 4. Calpain 1 but not Calpain 2 regulates activation of Bax in rd1 dying photoreceptors. (A, B) NIH3T3 cells were infected with retroviruses expressing shRNAs targeting Calpain 1 mRNA (Calpain 1 shRNA), shRNAs targeting Calpain 2 mRNA (Calpain 2 shRNA), or a retrovirus expressing an shRNA recognizing a scrambled sequence (scrambled shRNA). Western blotting for Calpain 1 (A) and Calpain 2 (B) was performed on total protein extracts from infected NIH3T3 cells and normalized with anti-actin antibodies (lower). (C) Downregulation of Calpain 1 mRNA by shRNA in rod-like cells in vitro differentiated from retinal neurospheres. Data represent percentages of positive cells labeled by immunofluorescence; $n=6$. Similar results were obtained with a second shRNA targeting a different region of Calpain 1 mRNA. (D) Downregulation of Calpain 2 mRNA by shRNA in rod-like cells in vitro differentiated from retinal neurospheres. Data represent percentages of positive cells labeled by immunofluorescence; $n=3$. Similar results were obtained with a second shRNA targeting a different region of Calpain 2 mRNA. (E) Representative images of data shown in (C). Calpain 1 is shown in green, and activated Bax is shown in red. Nuclear localization of Aif (red) and TUNEL staining (green) are indicated by arrows. Scale bar for all figure parts is shown at upper left and is 20 $\mu \mathrm{m}$.

Our previous studies showed that in vivo treatment with calpastatin, an inhibitor of calpain 1 and calpain 2, had protective effects in the $r d 1$ retina, ${ }^{4}$ thus suggesting that either calpain 1 or calpain 2 might be involved in activation of Bax. To define in more detail which calpain was involved in Bax activation, we downregulated expression of the two calpains in the cell culture model. We designed shRNAs that were specific to knock down either Calpain 1 or Calpain 2 as shown by Western blot analysis (Figs. 4A, 4B). Knockdown of calpain 1, known to be the Aif activating calpain, inhibited Bax activation, Aif nuclear translocation, and cell death (Figs. 4C, $4 \mathrm{E})$. Otherwise knockdown of Calpain 2 had no significant effect on Bax activation and cell death but could prevent caspase 12 nuclear translocation, known to be regulated by Calpain $2^{20}$ (Fig. 4D).
In vitro differentiated $r d 1$ mutant photoreceptors allowed us to determine that calpain 1 and cathepsin D are both involved in Bax activation during photoreceptor cell death, and that activated Bax is likely to promote Aif nuclear translocation as reported in other cell types. ${ }^{5-7}$

\section{Calpains Trigger Bax Activation in Three Models of Retinitis Pigmentosa}

We finally wanted to determine the interrelationship among these proteases and Bax and to understand if the molecular pathway unraveled in vitro was activated also in vivo in the degenerating retina. We injected in vivo $\mathrm{P} 10$ rd1 or $\mathrm{P} 9 \mathrm{P} 23 \mathrm{H}^{\mathrm{Tg}}$ or $\mathrm{P} 44 \mathrm{Rho}^{-1-}$ degenerating eyes with calpastatin, an inhibitor of calpains. We confirmed the ability of calpastatin to interfere with calpain activation (Figs. 5A-C, bottom; Supplementary Fig. S2). Calpastatin was not able to completely abolish calpain activity in $r d 1$ retinas, as we previously reported ${ }^{4}$; a similar result was observed in $\mathrm{P} 23 \mathrm{H}^{\mathrm{Tg}}$ retinas, but a significant reduction could be confirmed in both mutants (Student's $t$ test, $P \leq 0.001$ ) (Figs. 5A, 5B, bottom; Supplementary Fig. S2). Calpastatin was otherwise very efficient in blocking calpain activity of Rho ${ }^{-/-}$retinas (Fig. 5C, bottom; Supplementary Fig. S2). Interference with calpain activity using calpastatin was able to reduce Bax activation in the three models of RP (Figs. $5 \mathrm{~A}-\mathrm{C}$, top). Calpastatin was very efficient in blocking Bax in rd1 and $\mathrm{Rho}^{-/-}$mutant retinas but less efficient in $\mathrm{P} 23 \mathrm{H}^{\mathrm{Tg}}$ retinas, where a decreased but clear staining of activated Bax could be detected.

To determine if cathepsin $\mathrm{D}$ was downstream to calpain in vivo as shown in vitro, we analyzed cathepsin $\mathrm{D}$ activation by Western blotting in retinas after calpastatin treatment and found that blocking calpain activity significantly reduced cathepsin D activation in the treated retinas (Figs. 5D-G). Interference with cathepsin $\mathrm{D}$ activation was more prominent in the $r d 1$ and $\mathrm{Rho}^{--}$mutant retinas (Figs. 5D, 5F, 5G).

To confirm that Bax is downstream to calpains and plays an important role in cell death in the $r d 1$ mutant photoreceptors, we used the in vitro cell system of $r d 1$ mutant rod-like cells and downregulated Bax by shRNAs (Figs 5H, 5I). Downregulation of Bax interfered with cell death as defined by TUNEL staining, as well as Aif activation as defined by nuclear translocation of Aif (Fig. 5I). Bax knockdown did not affect calpain activation in in vitro differentiated $r d 1$ mutant rods (Fig. 5I).

Altogether these data support a model in which Bax is activated through calpains and cathepsin D in degenerating rod photoreceptors.

\section{Discussion}

In this study we demonstrated that Bax is activated in three different models of RP. The mechanism of photoreceptor cell death is still not well characterized, but some common pathways such as activation of calpains had been described while others appear to be specific to RP caused by particular mutations. ${ }^{11}$ Specifically, while apoptosis is normally associated with caspase activation and chromatin fragmentation, rod cell death is characterized by chromatin fragmentation but effector caspases are activated only in some models of RP. For instance, cell death in the $r d 1$ mutant retina is not much affected by caspase 3, while caspases appear to play an important role in retinas expressing mutant rhodopsin. ${ }^{10-12}$ Bax and Bak activation can lead to caspase initiation, but Bax mitochondrial localization does not necessarily trigger mitochondrial outer membrane permeabilization. ${ }^{2}$ Calpain 1 and Bax downregulation experiments in this study suggest that in the $r d 1$ mutant photoreceptors, Bax activation is otherwise 
A

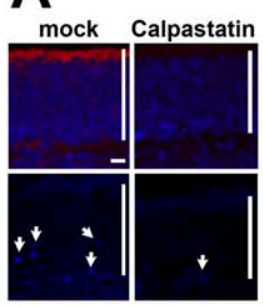

D

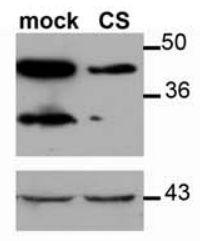

B

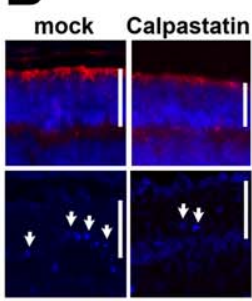

E

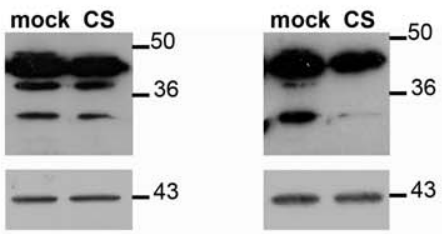

G

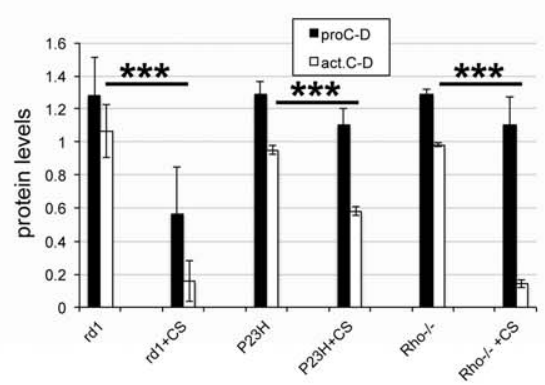

$\mathbf{H}$

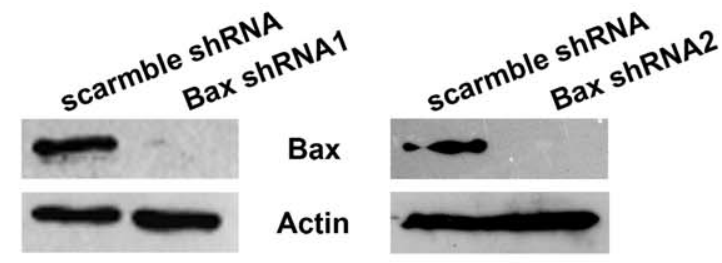

c
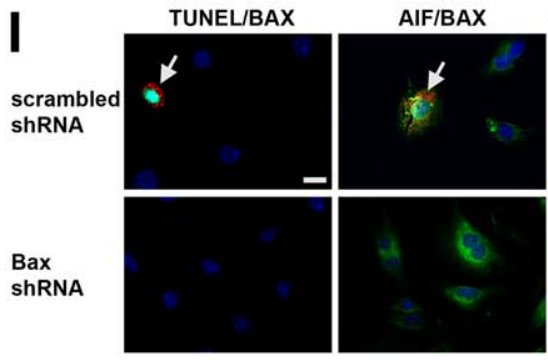

CALPAIN ACTIVITY

Bax

ShRNA
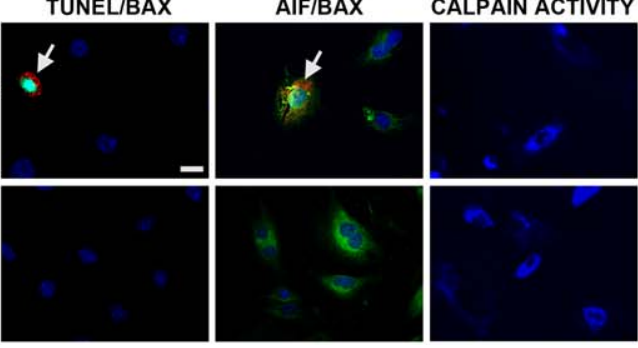

Figure 5. Calpains regulate activation of Bax and cathepsin $D$ in vivo. (A-C) Representative images of immunofluorescence analysis of retinas after mock intravitreal injection or injection of calpastatin in $\mathrm{P} 11$ rd 1 (A), $\mathrm{P} 10 \mathrm{P} 23 \mathrm{H}^{\mathrm{Tg}}(\mathbf{B})$, and $\mathrm{P} 45 \mathrm{Rho}^{-/-}(\mathbf{C})$ mice. Upper parts of figure are stained with an activated Bax-specific antibody (red), and nuclei are stained in blue with DAPI. Lower parts of figure are images of calpain activity assay (blue). Vertical white lines indicate the layer of photoreceptor cells; $n=3$. Scale bar for all figure parts is shown at upper left (A) and is $10 \mu \mathrm{m}$. (D-F) Western blotting of cathepsin D in

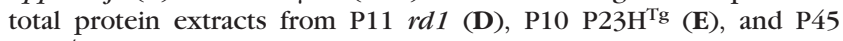
$\mathrm{Rho}^{-1-}$ (F) either mock injected or injected with calpastatin (CS). The antibody recognizes both cathepsin $\mathrm{D}(46 \mathrm{kDa})$ and activated cathepsin D (32 kDa). Normalization was performed with anti-actin antibodies (lower). Molecular weights are shown in kDa. (G) Quantification by densitometry of Western blotting shown in (D-F). A significant decrease of activated cathepsin D (white bars) was observed after injection of calpastatin (CS); $n=3$. Student's $t$-test, ${ }^{* * *} P \leq 0.001$ comparing activated cathepsin D in mock and calpastatin-treated

linked to Aif, a death activator that exits from the mitochondria and translocates to the nucleus, as described for other forms of neuronal degeneration. ${ }^{21}$

Our study indicates that the mechanisms by which Bax connects to retinal neurodamage in vivo requires two types of proteases. The molecular mechanisms of Bax activation have not been well characterized; but in this study we showed that in photoreceptor cells, Bax is activated by a protease cascade involving calpain 1 and cathepsin D. Bax needs to undergo conformational changes that can be promoted by several molecules, for example, tBid and cathepsin $\mathrm{D}$, and $\mathrm{pH}$ modifications. ${ }^{8,22}$ The mechanism of Bax activation by cathepsin $\mathrm{D}$ is still unknown, but cathepsin $\mathrm{D}$ has been suggested to possibly degrade a cytosolic chaperone that sequesters Bax. $^{8}$ We cannot exclude the possibility that in the retina $\mathrm{Bax}$ is influenced also by other factors such as changes in $\mathrm{pH}$. Tafani and colleagues ${ }^{22}$ showed that changes of intracellular $\mathrm{pH}$ interfered with Bax mitochondrial translocation. Changes of $\mathrm{pH}$ in the $r d 1$ mutant retina may be due to ceramide, which was previously found to induce intracellular alkalinization $^{23}$ and to be linked to cell death in retina degeneration Pde $6 b$ mutant mice. ${ }^{24}$ A similar mechanism was reported in glioma cells, where lysosomal accumulation of ceramide induced apoptosis mediated by cathepsin D activation and $\mathrm{Bax}$ insertion into the mitochondrial membrane. ${ }^{25}$ Loss of binding to prosurvival members of the $\mathrm{Bcl} 2$ family may also affect Bax activation, and we have shown that $\mathrm{Bcl} 2$ is reduced in two of the studied models and specifically the models with fastest degeneration. This observation suggests that the protease pathways may play a prevalent role in Bax activation in the degenerating retina but that $\mathrm{Bcl} 2$ downregulation may be required in more severe forms of degeneration.

The in vivo treatments with calpastatin confirmed that calpains regulate Bax activation through cathepsin D. The incomplete interference with Bax activation in the $\mathrm{P} 23 \mathrm{H}^{\mathrm{Tg}}$ retina could be explained by the failure of calpastatin to completely block calpain activity. A second explanation is the mechanism of cell death in $\mathrm{P} 23 \mathrm{H}^{\mathrm{Tg}}$ mice that involves calpain but also caspases, as previously reported; this may activate multiple pathways of Bax activation in this model of RP. ${ }^{11,12}$ We in fact observed correlation of cell death (TUNEL-positive cells) and calpain activity only in $50 \%$ of $\mathrm{P} 23 \mathrm{H}$ mutant photoreceptors. While calpastatin was able to strongly inhibit Bax activation in $r d 1$ mutant mice, it reduced but did not stop cell death. ${ }^{4}$ This observation suggests that in vivo inhibition of Bax may not be sufficient to completely block degeneration, as previously suggested in $r d 1$ mice in which Bax was knocked out. ${ }^{26}$

samples. (H) NIH3T3 cells were infected with retroviruses expressing two shRNAs targeting Bax mRNA (Bax shRNA) or with a retrovirus expressing an shRNA recognizing a scrambled sequence (scrambled shRNA). Western blotting was performed on total protein extracts from infected NIH3T3 cells and normalized with anti-actin antibodies (lower). (I) Downregulation of Bax mRNA by shRNA in rod-like cells in vitro differentiated from retinal neurospheres. Representative images of cells labeled by immunofluorescence; $n=3$. Similar results were obtained with a second shRNA targeting a different region of Bax mRNA. Activated Bax is shown in red; nuclear localization of Aif (green) and TUNEL staining (green) are indicated by arrows. DAPI staining of nuclei is shown in blue. Right: Calpain activity assay in which cells were exposed to a blue fluorescent calpain substrate visible in cells treated with a control shRNA as well as with an shRNA targeting Bax, suggesting that Bax activation does not affect calpain activity. No DAPI staining was performed on slides treated with calpain substrate. Scale bar for all parts of the figure is shown at upper left and is $20 \mu \mathrm{m}$. 
Finally, cathepsin D is likely to act downstream to calpains in experiments conducted in vitro, as its downregulation completely inhibited Bax activation in presence of active calpains. We could not test cathepsin $\mathrm{D}$ inhibition in vivo and address this question because treatment with pepstatin $\mathrm{A}$, an inhibitor of cathepsin D, had toxic effects when injected in the retina, as previously reported also by other researchers. ${ }^{9}$

In conclusion, we have demonstrated that Bax is activated during retinal degeneration in three models of RP and that its activation is regulated by calpains and cathepsin $\mathrm{D}$ in $r d 1$ and $\mathrm{Rho}^{-/-}$photoreceptors. Bax is only partially regulated by this cascade of proteases in dominant mutations of Rho, and other factors probably related to endoplasmic reticulum stress may affect Bax in this model of RP. Altogether these findings suggest that Bax activation should be evaluated when new therapeutic approaches are being developed to restrain retina degeneration.

\section{Acknowledgments}

The authors thank the Centro Servizi Stabulario Interdipartimentale of the University of Modena and Reggio Emilia for providing animal husbandry assistance, Thaddeus Peter Dryja for kindly providing the $\mathrm{P} 23 \mathrm{H}$ transgenic mouse line, and Carmine Spampanato and Diego Medina for reagents.

Supported by Fondazione Telethon (Grants GGP06096, GGP11201A), E-RARE 2009 (Comprehensive analysis of rod-cone photoreceptor degeneration associated with Rhodopsin gene mutations), and the Italian Ministry (Grants 2006053302_003, 20094CZ3M2).

Disclosure: A. Comitato, None; D. Sanges, None; A. Rossi, None; M.M. Humphries, None; V. Marigo, None

\section{References}

1. Willis SN, Adams JM. Life in the balance: how BH3-only proteins induce apoptosis. Curr Opin Cell Biol. 2005;17:617625 .

2. Renault TT, Teijido O, Antonsson B, Dejean LM, Manon S. Regulation of Bax mitochondrial localization by Bcl-2 and Bcl$\mathrm{x}(\mathrm{L})$ : keep your friends close but your enemies closer. Int $J$ Biochem Cell Biol. 2013;45:64-67.

3. Sanges D, Comitato A, Tammaro R, Marigo V. Apoptosis in retinal degeneration involves cross-talk between apoptosisinducing factor (AIF) and caspase-12 and is blocked by calpain inhibitors. Proc Natl Acad Sci U S A. 2006;103:17366-17371.

4. Paquet-Durand F, Sanges D, McCall J, et al. Photoreceptor rescue and toxicity induced by different calpain inhibitors. $J$ Neurochem. 2010;115:930-940.

5. Arnoult D, Parone P, Martinou JC, Antonsson B, Estaquier J, Ameisen JC. Mitochondrial release of apoptosis-inducing factor occurs downstream of cytochrome c release in response to several proapoptotic stimuli. J Cell Biol. 2002;159:923-929.

6. Moubarak RS, Yuste VJ, Greer PA, et al. Sequential activation of poly(ADP-ribose) polymerase 1, calpains, and Bax is essential in apoptosis-inducing factor-mediated programmed necrosis. Mol Cell Biol. 2007;27:4844-4862.

7. Polster BM, Basanez G, Etxebarria A, Hardwick JM, Nicholls DG. Calpain I induces cleavage and release of apoptosisinducing factor from isolated mitochondria. $\mathrm{J}$ Biol Chem. 2005;280:6447-6454.

8. Bidere N, Lorenzo HK, Carmona S, et al. Cathepsin D triggers Bax activation, resulting in selective apoptosis-inducing factor (AIF) relocation in $\mathrm{T}$ lymphocytes entering the early commitment phase to apoptosis. J Biol Chem. 2003;278:3140131411.
9. Doonan F, Donovan M, Cotter TG. Activation of multiple pathways during photoreceptor apoptosis in the rd mouse. Invest Ophthalmol Vis Sci. 2005;46:3530-3538.

10. Zeiss CJ, Neal J, Johnson EA. Caspase-3 in postnatal retinal development and degeneration. Invest Ophthalmol Vis Sci. 2004; 45:964-970.

11. Kaur J, Mencl S, Sahaboglu A, et al. Calpain and PARP activation during photoreceptor cell death in $\mathrm{P} 23 \mathrm{H}$ and S334ter rhodopsin mutant rats. PLoS One. 2011;6:e22181.

12. Gorbatyuk MS, Knox T, LaVail MM, et al. Restoration of visual function in $\mathrm{P} 23 \mathrm{H}$ rhodopsin transgenic rats by gene delivery of BiP/Grp78. Proc Natl Acad Sci U S A. 2010;107:5961-5966.

13. Olsson JE, Gordon JW, Pawlyk BS, et al. Transgenic mice with a rhodopsin mutation (Pro23His): a mouse model of autosomal dominant retinitis pigmentosa. Neuron. 1992;9:815-830.

14. Humphries MM, Rancourt D, Farrar GJ, et al. Retinopathy induced in mice by targeted disruption of the rhodopsin gene. Nat Genet. 1997;15:216-219.

15. Giordano F, De Marzo A, Vetrini F, Marigo V. Fibroblast growth factor and epidermal growth factor differently affect differentiation of murine retinal stem cells in vitro. Mol Vis. 2007;13: 1842-1850.

16. Demontis GC, Aruta C, Comitato A, De Marzo A, Marigo V. Functional and molecular characterization of rod-like cells from retinal stem cells derived from the adult ciliary epithelium. PLoS One. 2012;7:e33338.

17. Kim M, Jung SO, Park K, et al. Detection of Bax protein conformational change using a surface plasmon resonance imaging-based antibody chip. Biochem Biophys Res Commun. 2005;338:1834-1838.

18. Sizova OS, Shinde VM, Lenox A, Gorbatyuk MS. Modulation of cellular signaling pathways in $\mathrm{P} 23 \mathrm{H}$ rhodopsin photoreceptors. Cell Signal. 2014;26:665-672.

19. Jorgensen TN, McKee A, Wang M, et al. Bim and Bcl-2 mutually affect the expression of the other in T cells. J Immunol. 2007; 179:3417-3424.

20. Lee HJ, Lee SH, Park SH, et al. Purification of catalytically active caspase-12 and its biochemical characterization. Arch Biochem Biophys. 2010;502:68-73.

21. Cheung EC, Melanson-Drapeau L, Cregan SP, et al. Apoptosisinducing factor is a key factor in neuronal cell death propagated by BAX-dependent and BAX-independent mechanisms. J Neurosci. 2005;25:1324-1334.

22. Tafani M, Cohn JA, Karpinich NO, Rothman RJ, Russo MA, Farber JL. Regulation of intracellular $\mathrm{pH}$ mediates Bax activation in HeLa cells treated with staurosporine or tumor necrosis factor- $\alpha$. J Biol Chem. 2002;277:49569-49576.

23. Belaud-Rotureau MA, Leducq N, Macouillard Poulletier de Gannes F, et al. Early transitory rise in intracellular $\mathrm{pH}$ leads to Bax conformation change during ceramide-induced apoptosis. Apoptosis. 2000;5:551-560.

24. Strettoi E, Gargini C, Novelli E, et al. Inhibition of ceramide biosynthesis preserves photoreceptor structure and function in a mouse model of retinitis pigmentosa. Proc Natl Acad Sci U S A. 2010;107:18706-18711.

25. Dumitru CA, Sandalcioglu IE, Wagner M, Weller M, Gulbins E. Lysosomal ceramide mediates gemcitabine-induced death of glioma cells. J Mol Med. 2009;87:1123-1132.

26. Mosinger Ogilvie J, Deckwerth TL, Knudson CM, Korsmeyer SJ. Suppression of developmental retinal cell death but not of photoreceptor degeneration in Bax-deficient mice. Invest Ophthalmol Vis Sci. 1998;39:1713-1720. 